SLAC-PUB-8082

March 1999

\title{
Hierarchies Without Symmetries from Extra Dimensions
}

Nima Arkani-Hamed and Martin Schmaltz

Submitted toPhysical Review D

Stanford Linear Accelerator Center, Stanford University, Stanford, CA 94309

Work supported by Department of Energy contract DE-AC03-76SF00515. 
SLAC-PUB-8082

\title{
Hierarchies without Symmetries from Extra Dimensions
}

\author{
Nima Arkani-Hamed and Martin Schmaltz *
}

SLAC, Stanford University, Stanford, CA 94309

March 19, 1999

\begin{abstract}
It is commonly thought that small couplings in a low-energy theory, such as those needed for the fermion mass hierarchy or proton stability, must originate from symmetries in a high-energy theory. We show that this expectation is violated in theories where the Standard Model fields are confined to a thick wall in extra dimensions, with the fermions "stuck" at different points in the wall. Couplings between them are then suppressed due to the exponentially small overlaps of their wave functions. This provides a framework for understanding both the fermion mass hierarchy and proton stability without imposing symmetries, but rather in terms of higher dimensional geography. A model independent prediction of this scenario is non-universal couplings of the Standard Model fermions to the "Kaluza-Klein" excitations of the gauge fields. This allows a measurement of the fermion locations in the extra dimensions at the LHC or NLC if the wall thickness is close to the $\mathrm{TeV}$ scale.
\end{abstract}

\footnotetext{
*nima@slac.stanford.edu, schmaltz@slac.stanford.edu
} 


\section{Introduction}

The usual way of organizing our thinking about physics beyond the Standard Model (SM) is the effective field theory paradigm: all operators consistent with the symmetries are present in the theory, with higher-dimension operators suppressed by powers of the ultraviolet cutoff. The SM itself provides an exception to this expectation: the Yukawa couplings for all the fermions other than the top quark are much smaller than $\mathcal{O}(1)$. This does not lead to any fine-tuning problems since small Yukawa couplings are technically natural. Nevertheless, we are normally led to suspect that the fermion mass hierarchy is controlled by (weakly broken) flavor symmetries operative at shorter distances. Similar issues surround the question of proton decay in extensions submof the SM, especially when there is new physics at the TeV scale. Once again, some symmetry is normally invoked to forbid dangerous $1 /(\mathrm{TeV})$ suppressed interactions mediating proton decay. Furthermore, imposing global symmetries on low-energy effective theories, for instance, stabilizing the proton by declaring that the low-energy theory respects baryon number, is widely considered to be unsatisfactory given the lore that blackholes/wormholes violate all non-gauged symmetries. This seems particularly problematic for theories where the fundamental Planck scale is lowered close to the TeV scale $[1,2,3,4,5,6]$, and suggests that some sort of continuous $[3,7]$ or discrete [2] gauge symmetry is required to adequately suppress proton decay.

In this paper, we will show that all of this lore can easily and generically be violated in theories where the SM fields are constrained to live on a wall in $n$ extra dimensions, where gravity and perhaps other SM singlet fields are free to propagate. We will construct a simple model where our wall is

slightly thick in one of the extra dimensions. The wall will have interesting sub-structure: while the Higgs and SM gauge fields are free to propagate inside it, the SM fermion are "stuck" at different points in the wall, with wave functions given by narrow Gaussians as shown in Figure 1.

Without imposing any flavor symmetries on the short-distance theory, 


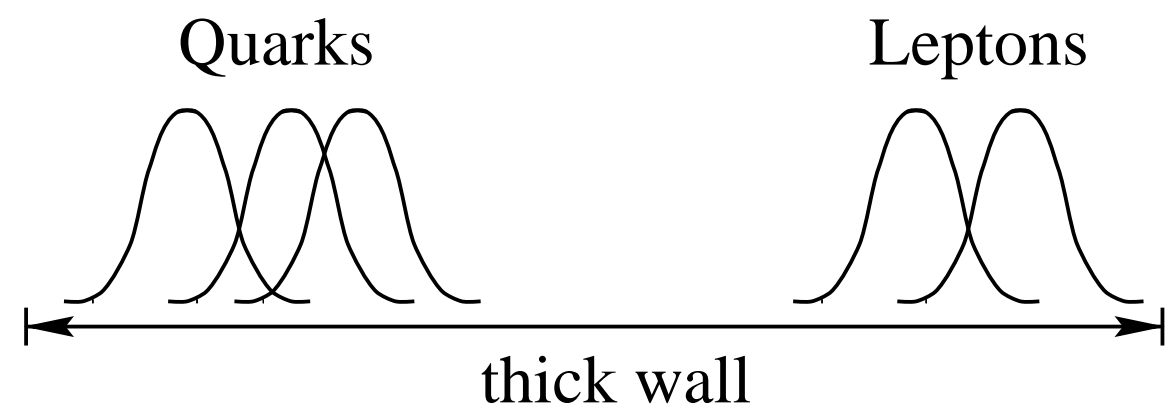

Figure 1: Profile of Standard Model fermion wave functions (vertical axis) in the extra dimensions (horizontal axis). The fermions freely propagate in $3+1$ dimensions (not shown) and are "stuck" at different locations in the extra dimensions. The gauge and Higgs fields' wave functions occupy the whole width of the thick wall. Direct couplings between the fermions are then suppressed by the exponentially small overlap of their wave functions. If - as shown here - quarks and leptons live on opposite ends of the wall profile protons become essentially stable. The hierarchy of Yukawa couplings arises from order one (in units of the fermion wave function width) distances between left and right handed components of the fermions.

we will see that the long-distance 4-dimensional theory can naturally have exponentially small Yukawa couplings, arising from the small overlap between left- and right-handed fermion wave functions. Similarly, without imposing any symmetries to protect against proton decay, the proton decay rate can be exponentially suppressed to safety if the quarks and leptons are are localized at different ends of the wall *. We emphasize that there is nothing fine-tuned about this from the point of view of the low-energy 4-dimensional theory; all the exponentially small couplings are technically natural. However, our examples violate the usual intuition that small couplings in a low-energy theory must be explained by symmetries in the high-energy theory. Instead,

* Our approach to to the fermion mass hierarchy similar in spirit to the one in [7]. For other approaches to suppressing Yukawa couplings and proton decay, see [6]. 
small couplings arise from the location and geometry of fermion fields stuck at different points in the extra dimensions, with no symmetries in the highenergy theory whatsoever. Note that this mechanism of separating fermions in an extra dimension is already being used to preserve chiral symmetry on the lattice in Kaplan's domain wall fermions [8]. Lattice simulations [9] show that chiral symmetry is protected very effectively by separating the left and right handed components of the fermions in the 5'th dimension.

If the wall thickness $L$ is close to the TeV scale, which is natural in theories with very low fundamental Planck scale, the mechanisms suggested in this paper can give rise to dramatic signals at future colliders. Since the SM gauge fields can only propagate inside the wall, $L$ effectively acts as the size of the extra dimensions for them ${ }^{\dagger}$. Therefore, at energies above $L^{-1}$, "KaluzaKlein" excitations (the higher harmonics of a particle in a box) of the gauge fields can be produced, and can scan the wall substructure. In particular, while the lowest excitation of the gauge fields (which we identify as the usual 4-d SM gauge fields) have a flat wave function throughout the wall and couple with standard strength to all the SM fermions, the KK excitations couple with non-universal strength to the fermions stuck at different points in the wall. For instance, if some of the fermions are stuck at special points (say the center of the wall), KK excitations of e.g. the photon can be baryophobic or leptophobic. More generally, measurements of the non-universal couplings of KK excitations to SM fermions can pin down their geometrical arrangement in the thick wall.

We emphasize that our prediction of non-universal couplings of the SM fermions to gauge and Higgs fields is model-independent, it only depends on the fact that the fermions are stuck at different points in the extra dimensions. Of course, the values of the different couplings are model-dependent and can

\footnotetext{
†Note that the dimensions where the gauge fields propagate need not be orthogonal to the large dimensions in which only gravity propagates; the gauge fields can just be restricted to live in a smaller part of the gravitational dimensions. The possibility of $\mathrm{TeV}$ sized extra dimensions with KK excitations for the SM gauge fields was first considered by Antoniadis [10]
} 
be used to distinguish between models.

In Section 2 we describe an explicit field theory mechanism which we use to construct a setup as outlined above; we discuss how to localize a single chiral fermion to defects in higher dimensions and then generalize to several fermions localized at different points in the vicinity of the same defect. In Section 3 we derive the exponentially small couplings which result from our framework and demonstrate how the scenario can explain the SM fermion mass hierarchy and suppress proton decay. We also comment on neutrino masses. Section 4 contains a brief discussion of experimental signatures resulting from the non-universal couplings of KK gauge fields. For example, our KK fields make a contribution to atomic parity violation with the correct sign to explain the discrepancies between the SM prediction and the most recent experimental results [11]. Our conclusions are drawn in section 5.

\section{Localizing chiral fermions}

\subsection{One chiral fermion in 5 dimensions}

For simplicity we limit ourselves to constructions with one extra dimension. Generalizations to higher dimensions are equally interesting and can be analyzed similarly. Localizing fields in the extra dimension necessitates breaking of higher dimensional translation invariance. This is accomplished in our construction of a thick wall by a spatially varying expectation value for a fivedimensional scalar field $\Phi$ as shown in Figure 2. We assume the expectation value to have the shape of a domain wall transverse to the extra dimension and centered at $x_{5}=0$. For example, such an expectation value could result from a $\mathbf{Z}_{2}$ symmetric potential for $\Phi$. $\ddagger$

We will now show that the Dirac equation for a five dimensional fermion in the background of this scalar field has a zero mode solution which corresponds

\footnotetext{
$\ddagger$ Interactions with the fermions below break this symmetry and render the domain wall profile unstable but the rate for tunneling to a constant expectation value can easily be suppressed to safety.
} 


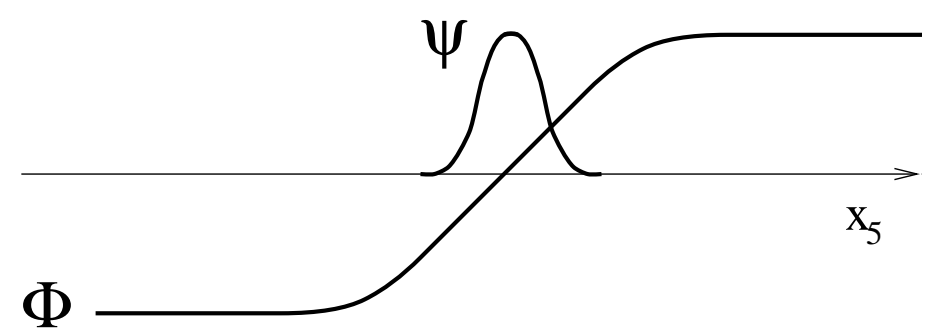

Figure 2: Profile of the scalar domain wall field $\Phi$ in the $x_{5}$ dimension. A chiral zero mode fermion is localized at the zero of $\Phi$.

to a four dimensional chiral fermion stuck at the zero of $\Phi$ [12]. A convenient representation for the $4 \times 4$ gamma matrices in five dimensions is

$$
\gamma^{i}=\left(\begin{array}{cc}
0 & \sigma^{i} \\
\bar{\sigma}^{i} & 0
\end{array}\right), \quad i=0 . .3, \quad \gamma^{5}=-i\left(\begin{array}{cc}
\mathbf{1} & 0 \\
0 & -\mathbf{1}
\end{array}\right) .
$$

As it will be useful in the following sections, we record below the two different Lorentz invariant fermion bilinears in 5 dimensions

$$
\bar{\Psi}_{1} \Psi_{2}, \quad \Psi_{1}^{T} C_{5} \Psi_{2}
$$

where

$$
C_{5}=\gamma^{0} \gamma^{2} \gamma^{5}=\left(\begin{array}{cc}
\epsilon & 0 \\
0 & -\epsilon
\end{array}\right) \quad \text { in the Weyl basis. }
$$

The first is the usual Dirac bilinear, while the second is the Majorana bilinear which generalizes the familiar 4-dimensional expression, where instead of $C_{5}$ we have $C_{4}=\gamma^{0} \gamma^{2}$.

The action for a five dimensional fermion $\Psi$ coupled to the background scalar $\Phi$ is then

$$
\mathcal{S}=\int \mathrm{d}^{4} \mathbf{x} \mathrm{d} x_{5} \bar{\Psi}\left[i \not \partial_{4}+i \gamma^{5} \partial_{5}+\Phi\left(x_{5}\right)\right] \Psi
$$

Here the coordinates of our $3+1$ dimensions are represented by $\mathbf{x}$ whereas the fifth coordinate is $x_{5}$; five-dimensional fields are denoted with capital 
letters whereas four-dimensional fields will be lower case. This Dirac operator is separable, and it is convenient to expand the $\Psi$ fields in a product basis

$$
\begin{gathered}
\Psi\left(\mathbf{x}, x_{5}\right)=\sum_{n}\left\langle x_{5} \mid L n\right\rangle P_{L} \psi_{n}(\mathbf{x})+\sum_{n}\left\langle x_{5} \mid R n\right\rangle P_{R} \psi_{n}(\mathbf{x}) \\
\bar{\Psi}\left(\mathbf{x}, x_{5}\right)=\sum_{n} \bar{\psi}_{n}(\mathbf{x}) P_{R}\left\langle L n \mid x_{5}\right\rangle+\sum_{n} \bar{\psi}_{n}(\mathbf{x}) P_{L}\left\langle R n \mid x_{5}\right\rangle,
\end{gathered}
$$

where the $\psi_{n}$ are arbitrary four-dimensional Dirac spinors and $P_{L, R}=(1 \pm$ $\left.i \gamma^{5}\right) / 2$ are chiral projection operators. We use a bra-ket notation for the eigenfunctions which diagonalize the $x_{5}$-dependent part of the Dirac operator; the kets $|L n\rangle$ and $|R n\rangle$ are solutions of

$$
\begin{array}{ll}
a a^{\dagger}|L n\rangle=\left(-\partial_{5}^{2}+\Phi^{2}+\dot{\Phi}\right) & |L n\rangle=\mu_{n}^{2}|L n\rangle \\
a^{\dagger} a|R n\rangle=\left(-\partial_{5}^{2}+\Phi^{2}-\dot{\Phi}\right) & |R n\rangle=\mu_{n}^{2}|R n\rangle,
\end{array}
$$

respectively. Here $\dot{\Phi} \equiv \partial_{5} \Phi$, and $a^{\dagger}$ and $a$ are "creation" and "annihilation" operators defined as

$$
\begin{array}{cc}
a & =\partial_{5}+\Phi\left(x_{5}\right) \\
a^{\dagger} & =\quad-\partial_{5}+\Phi\left(x_{5}\right) .
\end{array}
$$

The $|L n\rangle$ and $|R n\rangle$ each form an orthonormal set and for non-zero $\mu_{n}^{2}$ are related through $|R n\rangle=\left(1 / \mu_{n}\right) a|L n\rangle$ as can be verified easily from Eq.(7). The eigenfunctions with vanishing eigenvalues need not be paired however. It is no accident that we use simple harmonic oscillator (SHO) notation. For the special choice $\Phi\left(x_{5}\right)=2 \mu^{2} x_{5}$ the operators $a$ and $a^{\dagger}$ become the usual SHO creation and annihilation operators up to a normalization factor $\sqrt{2} \mu$, and the operator $a^{\dagger} a$ becomes the number operator $N$. The eigenkets are then related to the usual SHO kets by $|L n\rangle=|n\rangle$ and $|R n\rangle=|n-1\rangle$.

The pairing of eigenfunctions also persists for general $\Phi$. This follows most elegantly from considering the operators $Q=a \gamma^{0} P_{L}$ and $Q^{\dagger}=a^{\dagger} \gamma^{0} P_{R}$ which are the supercharges of an auxiliary supersymmetric quantum mechanics system [13] with Hamiltonian $H=\left\{Q, Q^{\dagger}\right\}$. Then $P_{L}|L n\rangle$ and $P_{R}|R n\rangle$ are 
the "boson" and "fermion" eigenstates of $H$ respectively, and the equality of eigenvalues of $|L n\rangle$ and $|R n\rangle$ is the usual boson-fermion degeneracy of supersymmetric theories. Again, zero modes need not be paired which allows us to obtain chiral 4-d theories. While most of what follows applies also to the case of general $\Phi$ we will find it convenient to use the SHO language.

Expanding in $|L n\rangle$ and $|R n\rangle$ the action for a 5-d Dirac fermion eq. (4) can be re-written in terms of a 4 -d action for an infinite number of fermions

$$
S=\int \mathrm{d}^{4} \mathbf{x}\left[\bar{\psi}_{L} i \not \partial_{4} P_{L} \psi_{L}+\bar{\psi}_{R} i \not \partial_{4} P_{R} \psi_{R}+\sum_{n=1}^{\infty} \bar{\psi}_{n}\left(i \not \partial_{4}+\mu_{n}\right) \psi_{n}\right] \text {. }
$$

The first two terms correspond to 4-d two-component chiral fermions, they arise from the zero modes of Eq.(7). The third term describes an infinite tower of Dirac fermions corresponding to the modes with non-zero $\mu_{n}$ in the expansion.

The zero mode wave functions are easily found by integrating $a^{\dagger}|L n\rangle=0$ and $a|R n\rangle=0$. The solutions

$$
\left\langle x_{5} \mid L, 0\right\rangle \sim \exp \left[-\int_{0}^{x_{5}} \Phi(s) \mathrm{d} s\right] \quad \text { and } \quad\left\langle x_{5} \mid R, 0\right\rangle \sim \exp \left[\int_{0}^{x_{5}} \Phi(s) \mathrm{d} s\right],
$$

are exponentials with support near the zeros of $\Phi$. In the infinite system that we are considering these modes cannot both be normalizable ${ }^{\S}$. It is easy to see that $|b, 0\rangle$ is normalizable if $\Phi(-\infty)<0$ and $\Phi(+\infty)>0$ as in Figure 2, and if $\Phi(-\infty)>0$ and $\Phi(+\infty)<0$ then the mode $|f, 0\rangle$ is normalizable. In the other cases there is no normalizable zero mode. For definiteness let us now specialize to the SHO. Then

$$
\left\langle x_{5} \mid L, 0\right\rangle=\frac{\mu^{1 / 2}}{(\pi / 2)^{1 / 4}} \exp \left[-\mu^{2} x_{5}^{2}\right],
$$

and $\left\langle x_{5} \mid R, 0\right\rangle$ is not normalizable. Thus the spectrum of four dimensional fields contains one left-handed chiral fermion in addition to an infinite tower

\footnotetext{
$\S$ Of course, we will be working in finite volume in the end, then the other mode is normalizable as well, but it is localized at the other end of the extra dimension. The existence of this other mode is dependent on boundary conditions.
} 
of massive Dirac fermions. The shape of the wave function of the chiral fermion is Gaussian, centered at $x_{5}=0$. Note that coupling $\Psi$ to $-\Phi$ would have rendered $\left\langle x_{5} \mid R, 0\right\rangle$ normalizable and we would have instead localized a massless right handed chiral fermion.

For clarity, let us write the full wave function of the massless chiral fermion in the chiral basis

$$
\Psi\left(\mathbf{x}, x_{5}\right)=\left(\begin{array}{c}
\left\langle x_{5} \mid L, 0\right\rangle \psi(\mathbf{x}) \\
0
\end{array}\right)
$$

\subsection{Many chiral fermions}

We can easily generalize Eq. (4) to the case of several fermion fields. We simply couple all 5-d Dirac fields to the same scalar $\Phi$

$$
\mathcal{S}=\int \mathrm{d}^{5} x \sum_{i, j} \bar{\Psi}_{i}\left[i \not \partial_{5}+\lambda \Phi\left(x_{5}\right)-m\right]_{i j} \Psi_{j} .
$$

Here we allowed for general Yukawa couplings $\lambda_{i j}$ and also included masses $m_{i j}$ for the fermion fields. Mass terms for the five-dimensional fields are allowed by all the symmetries and should therefore be present in the Lagrangian. In the case that we will eventually be interested in - the standard model - the fermions carry gauge charges. This forces the couplings $\lambda_{i j}$ and $m_{i j}$ to be block-diagonal, with mixing only between fields with identical gauge quantum numbers. For simplicity we will set $\lambda_{i j}=\delta_{i j}$ in this paper, then $m_{i j}$ can be diagonalized with eigenvalues $m_{i}$.

Finding the massless four-dimensional fields is completely analogous to the single fermion case of the last section. Each 5 -d fermion $\Psi_{i}$ gives rise to a single 4-d left chiral fermion. Again, the wave functions in the 5th coordinate are Gaussian, but they are now centered around the zeros of $\Phi-m_{i}$. In the SHO approximation this is at $x_{5}^{i}=m_{i} / 2 \mu^{2}$. Thus, at energies well below $\mu$ the five-dimensional action above describes a set of non-interacting four dimensional chiral fermions localized at different 4-d "slices" in the 5th dimension. Note that while the overall position of the massless fermions 
in the $x_{5}$-direction is a dynamical variable (the location of the zero of $\Phi$ ), the relative positions of the various fermions are fixed by the $m_{i}$. Thus even when we turn on interactions between the massless fields, the relative distances which control the size of coupling constants in the effective 4-d theory stay fixed.

We now exhibit the field content of the 5-d theory which can reproduce the chiral spectrum of the 4-d SM as localized zero modes. First note that by choosing all $\lambda$ 's positive we have localized only left handed chiral Weyl spinors. That implies that we will construct the SM using only left handed spinors, the right handed fields are represented by their charge conjugates $\bar{\psi}^{c}$. Then the SM arises simply by choosing 5 -d Dirac spinors $\left(Q, U^{c}, D^{c}, L, E^{c}\right)$ transforming like the left-handed SM Weyl fermions $\left(q, u^{c}, d^{c}, l, e^{c}\right)$.

We also briefly mention how we imagine confining gauge fields to a $(3+1)-$ dimensional wall. A field-theoretic mechanism for localizing gauge fields was proposed by Dvali and Shifman and was later extended and applied in [1] (see also [15]). The idea is to arrange for the gauge group to confine outside the wall; the flux lines of any electric sources turned on inside the wall will then be repelled by the confining regions outside and forced to propagate only inside the wall. This traps a massless gauge field on the wall. Since the gauge field is prevented to enter the confined region, the thickness $L$ of the wall acts effectively as the size of the extra dimensions in which the gauge fields can propagate. Notice that in a picture like this, the gauge couplings will exhibit power law running above the scale $L^{-1}$, and so the scenario of

[6] for gauge coupling unification may be implemented, without the presence of any new dimensions beyond the large gravitational dimensions.

\section{Exponentially small 4 -d couplings}

In this section we present two examples of applications for our central result: exponentially small couplings from small wave function overlaps of fields which are separated in the fifth dimension. The two examples we consider are 
SM Yukawa couplings and proton decay. Since our exponential suppression factors dominate any power suppression we will not keep track of the various powers of scales which arise from matching 5-d to 4-d Lagrangians.

\subsection{Yukawa couplings}

In this section we apply our mechanism to generating hierarchical Yukawa couplings in four dimensions. Concentrating on only one generation and the lepton sector for the moment, we start with the five-dimensional fermion fields with action

$$
\mathcal{S}=\int \mathrm{d}^{5} x \bar{L}\left[i \not \partial_{5}+\Phi\left(x_{5}\right)\right] L+\bar{E}^{c}\left[i \not \partial_{5}+\Phi\left(x_{5}\right)-m\right] E^{c}+\kappa H L^{T} C_{5} E^{c} .
$$

where $C_{5}$ was defined in Eq. (3). As discussed in the previous sections, we find a left-handed massless fermions $l$ from $L$ localized at $x_{5}=0$ and $e^{c}$ from $E^{c}$ localized at $x_{5}=r \equiv m /\left(2 \mu^{2}\right)$. For simplicity, we will assume that the Higgs is delocalized inside the wall. We now determine what effective fourdimensional interactions between the light fields results from the Yukawa coupling in eq. (14). To this end we expand $L$ and $E^{c}$ as in eq. (6) and replace the Higgs field $H$ by its lowest Kaluza-Klein mode which has an $x_{5}$-independent wave function. We obtain for the Yukawa coupling

$$
\mathcal{S}_{Y u k}=\int \mathrm{d}^{4} \mathbf{x} \kappa h(\mathbf{x}) l(\mathbf{x}) e^{c}(\mathbf{x}) \int \mathrm{d} x_{5} \phi_{l}\left(x_{5}\right) \phi_{e^{c}}\left(x_{5}\right) .
$$

Here $\phi_{l}\left(x_{5}\right)$ and $\phi_{e^{c}}\left(x_{5}\right)$ are the zero-mode wave functions for the lepton doublet and singlet respectively. $\phi_{l}$ is a Gaussian centered at $x_{5}=0$ whereas

$\phi_{e^{c}}$ is centered at $x_{5}=r$. The overlap of Gaussians is itself a Gaussian and we find

$$
\int \mathrm{d} x_{5} \phi_{l}\left(x_{5}\right) \phi_{e^{c}}\left(x_{5}\right)=\frac{\sqrt{2} \mu}{\sqrt{\pi}} \int \mathrm{d} x_{5} e^{-\mu^{2} x_{5}^{2}} e^{-\mu^{2}\left(x_{5}-r\right)^{2}}=e^{-\mu^{2} r^{2} / 2} .
$$

This result is in agreement with the intuitive expectation from Figure 2. Any coupling between the two chiral fermions is necessarily exponentially 


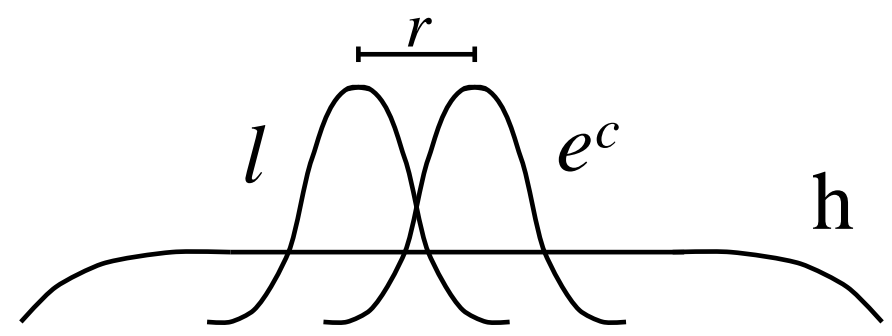

Figure 3: Yukawa coupling: the Gaussian wave functions of the fermions $l$ and $e^{c}$ overlap only in an exponentially small region, suppressing the effective Yukawa coupling exponentially.

suppressed because the two fields are separated in space. The coupling is then proportional to the exponentially small overlap of the wave functions.

Note that we did not impose any chiral symmetries in the fundamental theory to obtain this result: the coupling $\kappa$ can violate the electron chiral symmetry by $O(1)$. Even with chiral symmetry maximally broken in the fundamental theory, we obtain an approximate chiral symmetry in the low energy, 4-d effective theory.

\subsection{Long live the proton}

Proton decay places a very stringent constraint on most extensions of the standard model. Unless a symmetry can be imposed to forbid either baryon or lepton number violation, proton decay forces the scale of new physics to be extremely high. In particular one might be tempted to conclude that proton decay kills all attempts to lower the fundamental Planck scale $M_{*}$ significantly beneath the GUT scale, unless continuous or discrete gauge symmetries are invoked. We now show that these no-go theorems are very elegantly evaded by separating wave functions in the extra dimensions. Consider for simplicity a one-generation model in five dimensions where the standard model fermions are again localized in the $x_{5}$ direction by coupling the five-dimensional fields to the domain wall scalar $\Phi$. Assume that all quark fields are localized near $x_{5}=0$ whereas the leptons are near $x_{5}=r$ as de- 
picted schematically in Figure 1. We allow the five-dimensional theory to violate both baryon number and lepton number maximally, and we assume that we can parameterize this violation by local operators $\$$. Then we can expect the following dangerous looking five-dimensional baryon and lepton number violating operators

$$
\mathcal{S} \sim \int \mathrm{d}^{5} x \frac{\left(Q^{T} C_{5} L\right)^{\dagger}\left(U^{c T} C_{5} D^{c}\right)}{M_{*}^{3}}
$$

To obtain the corresponding four-dimensional proton decay operator we simply replace the five-dimensional fields by the zero mode fields and calculate the wave function overlap in $x_{5}$. The result is

$$
\mathcal{S} \sim \int \mathrm{d}^{4} \mathbf{x} \delta \times \frac{(q l)^{\dagger}\left(u^{c} d^{c}\right)}{M_{*}^{2}}
$$

where

$$
\delta \sim \int \mathrm{d} x_{5}\left[e^{-\mu^{2} x_{5}^{2}}\right]^{3} e^{-\mu^{2}\left(x_{5}-r\right)^{2}} \sim e^{-3 / 4 \mu^{2} r^{2}} .
$$

Already for a separation of $\mu r=10$ we obtain $\delta \sim 10^{-33}$ which renders these operators completely safe even for $M_{*} \sim 1 \mathrm{TeV}$. Thus we imagine a picture where quarks and leptons are localized near opposite ends of the wall so that $r \sim L$. Once again, even if baryon and lepton number are maximally broken in the 5-d theory at short distances, the coupling generated in the 4-d theory is exponentially suppressed and can be harmless.

In the following subsection, we present an alternate way of understanding the suppression of proton decay which also shows that corrections to this picture, either coming from quantum loops or exchange of new degrees of freedom, can be harmless.

\subsection{Long live the proton, again}

There is an alternative way of understanding the $e^{-(\mu r)^{2}}$ suppression which is physically transparent and shows that all radiative corrections are also

\footnotetext{
INon-local operators which result from integrating out massive bulk fields are discussed in the next subsection.
} 
suppressed by the same exponential factor. Even though it applies equally well to the case of Yukawa couplings we will only describe the analysis for proton decay here. In order to decay the proton using the local $Q Q Q L$ interaction, the quarks and leptons must propagate into the bulk of the wall, away from the points where they are massless (see Fig 4). Because e.g. the

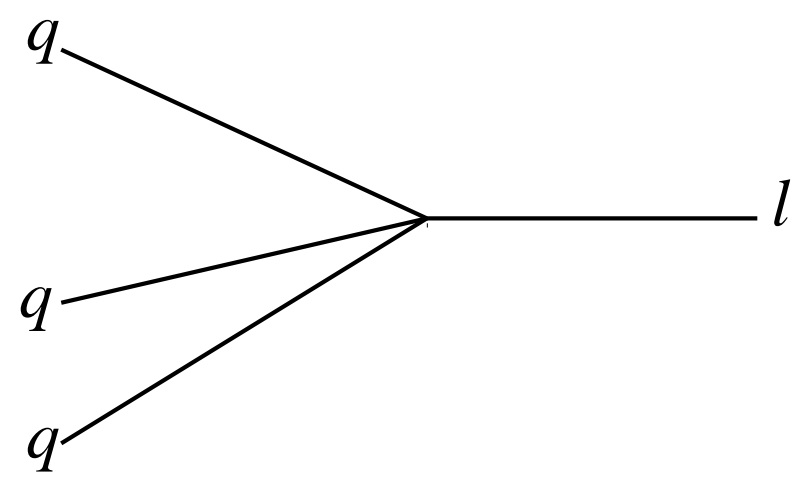

Figure 4: Tree-level proton decay diagram, drawn in position space for the fifth dimension. The quarks stuck at one end of the wall and the lepton stuck at the other end propagate to some interior point s where they interact via the higher-dimensional QQQL operator. The "free" propagator to go to a point in the bulk is given by the value of the (Gaussian) zero-mode wave function at that point.

quarks are getting more massive as they move into the bulk, the propagator from the plane where they live into the bulk is suppressed. Intuitively, for each slice between $x_{5}$ and $x_{5}+\delta x_{5}$, a Yukawa propagator $e^{-m\left(x_{5}\right) \delta x_{5}}$ must be paid. Therefore, the propagator to reach a final point $x_{*}$ is proportional to

$$
\prod_{\text {slices }} e^{-m\left(x_{5}\right) \delta x_{5}}=e^{-\int^{x_{*}} m\left(x_{5}\right) d x_{5}}=e^{-\mu^{2} x_{*}^{2}}
$$

for $m\left(x_{5}\right)=2 \mu^{2} x_{5}$. This is exactly the wave function for the zero mode evaluated at $x_{*}$, as is intuitively expected and can also be seen more formally. In order to evaluate the tree level diagram of Figure 4, we have to integrate over the interaction vertex, yielding for the coefficient of the proton decay 
operator

$$
\delta \sim \int d x_{5}\left(\phi_{q}\left(x_{5}\right)\right)^{3} \phi_{l}\left(x_{5}\right)
$$

precisely reproducing the result from our earlier "overlap of wave functions" picture. This approach also makes clear why higher order corrections do not significantly change the result. Indeed, the most general diagram for proton decay takes the form of Figure 5: the effect of all interactions are encoded in a modified propagator into the bulk and modified interaction vertex. The modified propagator has the simple interpretation of being the wave function of the zero mode in the interacting theory. The exact form of the modified vertex is unknown. However, the vertex will still be point-like on scales of order $\mu$, because all the interactions modifying it are mediated by particles of mass $\mu$, which can only smear the vertex on scales of order $\geq \mu$. Since the propagators involved are needed at distances $L \sim 10 \mu^{-1}$, the vertex in the Figure 5 is still effectively point-like, and so the picture of the suppression of proton decay through exponentially small wave function overlaps persists, if we replace the (free) Gaussian zero-mode wave functions by the true interacting ones.

So far we have considered proton decay operators induced by shortdistance physics above the cutoff $M_{*}$; but what about effects coming from integrating out fields possibly lighter than $M_{*}$ ? In particular, we may worry that while the separation of quarks and leptons suppresses higher-dimensional operators linking them, operators involving only quarks on one side and violating baryon number, or leptons on the other side violating lepton number, are not suppressed. If a light field of mass $m$ freely propagates inside the wall, this may induce operators violating both $B$ and $L$ suppressed only by $e^{-m L}$ (see Figure 6). However, in order to specifically induce proton decay, this light field would have to be fermionic. In particular, no gauge or Higgs boson exchanges can ever give rise to proton decay. If we make the single assumption that all delocalized fermions have masses of order the cutoff $M_{*}$, then their exchange can at most give $e^{-M_{*} L}$ contributions which are comparable to the $e^{-(\mu L)^{2}}$ effects we have considered. Note that this argument 


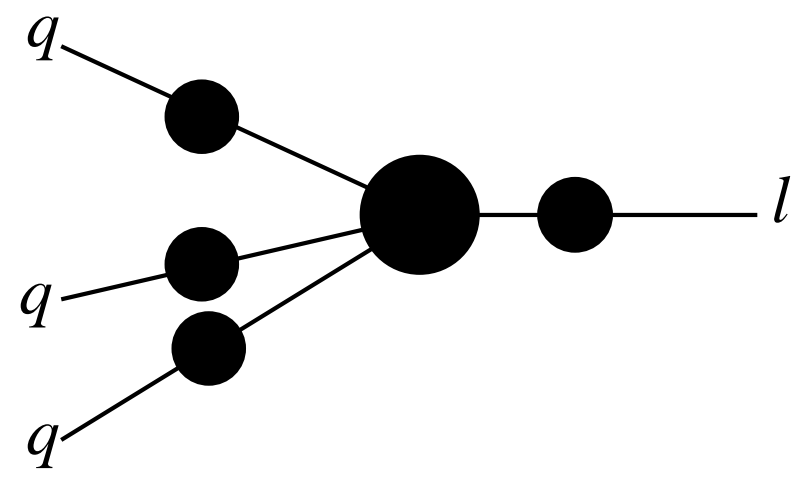

Figure 5: General proton decay diagram including higher order effects. The blobs on propagators denotes the all-order propagators, and the blob on the vertex denotes the corrected vertex. The corrected propagator is nothing other than the corrected zero-mode wave function. The corrected vertex is still local on scales larger than the width of the fermion wave function. This diagram is therefore well-approximated by the overlap between the corrected wave functions of the quark and lepton zero modes, which gives the enormous suppression of proton decay.

implies that grand unification at a scale as low as $\mu$ or $M_{*}$ does not lead to rapid proton decay, as long as there are no delocalized fermionic fields with masses below $M_{*}$. We cannot resist the temptation to speculate that the same vacuum expectation values (VEVs) which break the GUT symmetry near the scale $M_{*}$ may also be responsible for the separation of the SM fermions in the 5'th dimension. For example the $m_{i j}$ of Eq. (13) could stem from the VEV of a GUT symmetry breaking field which points in the (B-L) direction. Then the SM fermions would be split according to their baryon and lepton numbers. A VEV in the hypercharge direction would arrange the fermions according to their hypercharge.

We have seen that without imposing any symmetries on the underlying theory, proton decay can be adequately suppressed if quarks and leptons are stuck at different points in extra dimensions. One might then wonder what happened to the general lore that black-hole/wormhole effects violate all non-gauged symmetries and are therefore dangerous. In evaluating this 


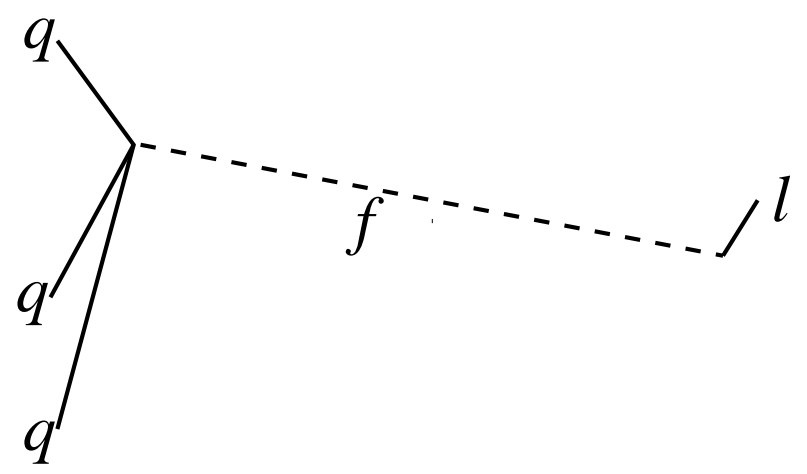

Figure 6: The exponential suppression of proton decay through small wave function overlaps can be avoided if there is a delocalized light fermion $f$ with baryon and lepton number violating couplings as seen above. This amplitude is only suppressed by the Yukawa factor $e^{-m_{f} L}$. In order to avoid too large a rate for proton decay without imposing any symmetries, we must postulate that there are no delocalized fermions lighter than $M_{*}$.

argument, we have to recall that it was Planck-scale sized wormholes giving the supposedly $O(1)$ symmetry violating effects. Since these have a mass above the cutoff, all their effects can be encoded in terms of local operators suppressed by $M_{p} \geq M_{*}$; and indeed, we presumed that such "dangerous" operators were really present in the theory. However, their effects are harmless because the quarks and leptons are stuck at different points, yet have to be dragged close to each other for the dangerous operators to be operative. Of course, in the effective theory at distances longer than $L$, the quarks and leptons look like they are on top of each other, so the above suppression mechanism does not seem to apply. However, only wormholes larger than $L$ are admissible in this effective theory, and any effect they induce will be exponentially suppressed by their action

$$
e^{-S}, \quad S \sim \int d^{4+n} x M_{p}^{(2+n)} \mathcal{R} \sim\left(M_{p} L\right)^{(2+n)}
$$

which is a far larger suppression than the effects we have computed $\sim$ $e^{-(\mu L)^{2}} \sim e^{-M_{*} L}$. The largest possible effect which might arise from wormholes would come from long and skinny wormholes which stretch from the 
proton to the lepton; but even these are completely safe as their action is at least $\sim e^{-\left(M_{p} L\right)}$.

\subsection{Neutrino masses}

Separating quarks and leptons at different points in extra dimensions can easily suppress proton decay without imposing any symmetries on the highenergy theory. On the other hand, as already mentioned, operators violating baryon and lepton number need not be suppressed. In fact, in the absence of any symmetries in the high-energy theory, a Majorana neutrino mass operator

$$
\mathcal{S} \sim \int d^{5} x \frac{L^{T} C_{5} L H^{*} H^{*}}{M_{*}^{2}}
$$

turns into an unsuppressed Majorana mass term for the 4-d zero mode

$$
\mathcal{S} \sim \int d^{4} \mathbf{x} \frac{l l h^{*} h^{*}}{M_{*}}
$$

since there is no small overlap between l's wave function with itself. There are a number of ways of resolving this problem; we will just mention the obvious strategy of adding a right-handed neutrino and gauging $(B-L)$. Of course $(B-L)$ must be broken in such a way as to not allow large Majorana masses after breaking. In our framework this would be most naturally achieved with a $(B-L)$ breaking VEV which is localized within the wall but at some distance from the lepton field $l$ so that the Majorana neutrino mass is exponentially suppressed ॥. In addition one would also get small Dirac neutrino masses, with the tiny Yukawa couplings originating from the overlap between right- and left- handed neutrino wave functions.

\footnotetext{
" $(B-L)$ could also be broken everywhere within the wall if a discrete subgroup remains preserved, or it could be broken on a distant wall if $(B-L)$ is gauged in the large bulk where gravity propagates $[3,7]$. For another approach to neutrino masses see [16].
} 


\subsection{Summary of scales}

Let us close by giving an account of the various scales we are now imagining. Recall that the at the edge of the wall, the fermion mass $\langle\Phi\rangle$ is $\sim \mu^{2} L \sim 10 \mu$, and must not be larger than the ultraviolet cutoff $M_{*}$. In fact we will take them to be comparable. Therefore, we have three scales in the problem: the UV cutoff $M_{*}, \mu$ and the wall thickness scale $L^{-1}$, with magnitudes related roughly as

$$
M_{*} \sim 10 \mu \sim 100 L^{-1}
$$

Since we cannot push $L^{-1}$ significantly below $\sim \mathrm{TeV}$, the fundamental scale $M_{*}$ is bounded below by $\sim 100 \mathrm{TeV}$. This is actually desirable from another point of view: in the absence of flavor symmetries, it is difficult to protect against flavor changing neutral currents without pushing the scale of higherdimension operators to $\sim 100 \mathrm{TeV}$.

Notice that even though the theory becomes effectively 5-dimensional above $L^{-1}$, the theory is perturbative up to the UV cutoff $M_{*}$. From the 4-dimensional viewpoint, we have $N_{K K} \sim\left(M_{*} L\right) / 2 \pi \sim 10-100$ gauge and Higgs field KK modes, and so the effective expansion parameter is

$$
\frac{h_{4}^{2}}{16 \pi^{2}} \times N_{K K} \sim O(1)
$$

where $h_{4}$ is a generic low-energy gauge coupling or top Yukawa coupling. From the higher-dimensional point of view, the theory is on the edge of being strongly coupled at the UV cutoff $M_{*}$.

Finally, we wish to give a rough idea of the sort of suppressions which are generated by our $e^{-(c \mu L)^{2}}$ size effects, with $c=1 / 2$ for Yukawa couplings and $c=3 / 4$ for proton decay:

\begin{tabular}{c|cccccccc}
$\mu r$ & 0 & 1 & 2 & 3 & 4 & 5 & 7 & 10 \\
\hline $\exp \left(-c \mu^{2} r^{2}\right)$ & 1 & 1 & $10^{-1}$ & $10^{-2}$ & $10^{-4}$ & $10^{-6}$ & $10^{-11}$ & $10^{-33}$ \\
\hline & & $\lambda_{t}$ & & $\cdots$ & & $\lambda_{e}$ & $\cdots$ & $\Gamma_{\text {proton }}$
\end{tabular}

It is attractive that for $\mu r$ just ranging between $1-10$, we can get appropriate sizes for everything from the top Yukawa coupling $\lambda_{t}$ (for $\mu r \sim 1$ ), to 
the electron Yukawa $\lambda_{e}(\mu r \sim 5)$, to sufficient suppression for proton decay $(\mu r \sim 10)$.

\section{Cartography with gauge fields}

While the SM fermion fields are stuck at different points in the extra dimension, the gauge fields are totally delocalized, and we expect that we can probe the locations of the fermions using the gauge fields. Cartography of the SM fermions with gauge fields will become an experimental science to be performed at the LHC or NLC if the wall thickness is as large as $\sim 1 \mathrm{TeV}^{-1}$.

To see how this works explicitly, consider a toy example with two 4d chiral fermions $\psi_{1}, \psi_{2}$, transforming identically under a gauge group $G$, but stuck to different points $s_{1}, s_{2}$ in the extra dimensions. At distances larger than the width of their wavefunction in the extra dimensions, the dynamics that localizes the fermions is irrelevant; we can approximate their wavefunctions as delta functions or, what is the same, fix them to live on a 3 dimensional wall, while gauge fields freely propagate in the bulk. The effective coupling to gauge fields is fixed by gauge invariance to be

$$
\mathcal{S} \sim \int d^{4} \mathbf{x} \bar{\psi}_{1} \bar{\sigma}^{\mu} T^{a} \psi_{1} A_{\mu}^{a}\left(\mathbf{x}, s_{1}\right)+\bar{\psi}_{2} \bar{\sigma}^{\mu} \psi_{2} A_{\mu}\left(\mathbf{x}, s_{2}\right)
$$

Let us Fourier expand $A_{\mu}$ as

$$
A_{\mu}\left(\mathbf{x}, x_{5}\right)=\frac{A_{\mu}^{(0)}(\mathbf{x})}{\sqrt{2}}+\sum_{n=1}^{\infty} A_{\mu}^{(n)}(\mathbf{x}) \cos \left(k_{n} x_{5}\right)+\sum_{n=1}^{\infty} B_{\mu}^{(n)}(\mathbf{x}) \sin \left(k_{n} x_{5}\right),
$$

where $k_{n}=\frac{2 \pi}{L} n$. Here $A_{\mu}^{(0)}$ corresponds to the massless 4-d gauge field, and the $A_{\mu}^{(n)}$ and $B_{\mu}^{(n)}$ are KK excitations with masses $k_{n}$. Inserting this expansion into Eq. (27) we find the effective couplings of the tower of 4-d KK gauge fields to the fermions. Note that since $A_{\mu}^{(0)}$ has a flat wave function in the extra dimensions, it has the same coupling to both fermions as required by 4-dimensional gauge invariance. However the couplings of the fermions to 
the massive KK states, $A$ and $B$, are proportional to cosines and sines from the wave functions of the KK states at the locations of the fermions **.

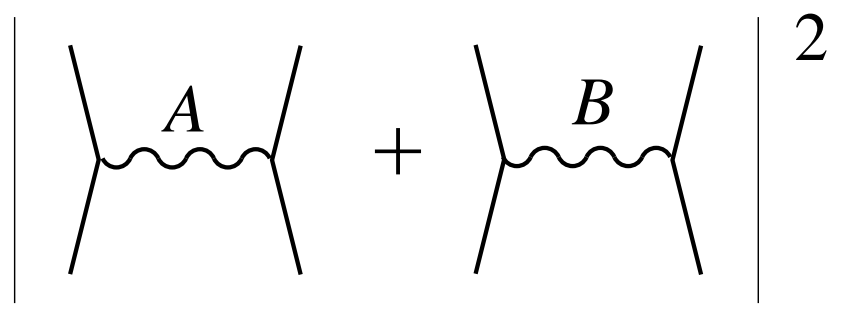

Figure 7: KK exchange diagrams.

Suppose that we are sitting on the $s$-channel resonance for particleantiparticle annihilation mediated by the $n$ 'th KK modes of $A$ and $B$. Then the relative cross section for $1^{+} 1^{-} \rightarrow 1^{+} 1^{-}$(and $2^{+} 2^{-} \rightarrow 2^{+} 2^{-}$) as calculated from the diagrams in Figure 7 will be different from $1^{+} 1^{-} \rightarrow 2^{+} 2^{-}$:

$$
\begin{aligned}
& \sigma\left(1^{+} 1^{-} \rightarrow A^{(n)}, B^{(n)} \rightarrow 1^{+} 1^{-}\right) \equiv \sigma_{n} \\
& \sigma\left(2^{+} 2^{-} \rightarrow A^{(n)}, B^{(n)} \rightarrow 2^{+} 2^{-}\right)=\sigma_{n} \\
& \sigma\left(1^{+} 1^{-} \rightarrow A^{(n)}, B^{(n)} \rightarrow 2^{+} 2^{-}\right)=\sigma_{n} \cos ^{2}\left(k_{n}\left(s_{1}-s_{2}\right)\right)
\end{aligned}
$$

and this can be used to gain information on the distance between $\psi_{1}, \psi_{2}$ in the extra dimensions.

Actually, there are subtleties in this analysis associated with the mechanism for localizing gauge fields. For example, in the Dvali-Shifman mechanism, confinement outside the wall forces specific boundary conditions $F_{\mu 5}=$ 0 at the edges of the wall [15]. This then enforces $\partial_{5} A_{\mu}\left(\mathbf{x}, x_{5}\right)=0$ at $x_{5}=0, L$, and the $B^{(n)}$ are eliminated. In addition, the $A^{(n)}$ may now be periodic or anti-periodic, thus $k_{n}=\frac{\pi}{L} n$. The position dependence of KK gauge couplings raises the interesting possibility that some of the KK excitations of various fields may be leptophobic or baryophobic if the quarks

\footnotetext{
${ }^{* *}$ Note that these couplings are valid for the KK modes with wavelength long compared to the fermion localization width; shorter wavelength KK modes can resolve the fermion wave function and so the delta function approximation for the fermion wave function is inadequate.
} 
or leptons sit at nodes of their wave functions. More generally, the cross sections above are modified to

$$
\begin{aligned}
& \sigma\left(1^{+} 1^{-} \rightarrow A^{(n)} \rightarrow 1^{+} 1^{-}\right)=\sigma_{n} \cos ^{4}\left(k_{n} s_{1}\right) \\
& \sigma\left(2^{+} 2^{-} \rightarrow A^{(n)} \rightarrow 2^{+} 2^{-}\right)=\sigma_{n} \cos ^{4}\left(k_{n} s_{2}\right) \\
& \sigma\left(1^{+} 1^{-} \rightarrow A^{(n)} \rightarrow 2^{+} 2^{-}\right)=\sigma_{n} \cos ^{2}\left(k_{n} s_{1}\right) \cos ^{2}\left(k_{n} s_{2}\right)
\end{aligned}
$$

There are clearly other interesting possibilities arising from the nonuniversal couplings of SM fermions to the KK excitations. As one example, in our scenario for suppressing proton decay by separating quark and lepton wave functions, the non-standard coupling of the quarks and leptons to the KK modes has an interesting impact on atomic parity violation (APV). The latest experimental results [11] indicate that the measured weak charge of the nucleus is lower than the SM expectation by $\sim 2.5 \sigma$. If we had a conventional Kaluza-Klein tower at $\sim \mathrm{TeV}$ scale, with standard couplings to quarks and leptons, this would enhance the SM contribution to APV. In our case, however, the situation can be different. If we impose the $F_{\mu 5}=0$ boundary conditions as stated above, then the first Kaluza-Klein excitation has the profile shown in Figure 8. Notice that the product of the quark and lepton couplings to the first KK excitation has the opposite sign as in the $\mathrm{SM}$, and gives a contribution to atomic parity violation that moves in the right direction. The sign of this effect is an inevitable consequence of our mechanism for suppressing proton decay, and the correct magnitude can be obtained if the wall thickness is $\sim 1 \mathrm{TeV}$.

\section{Conclusions}

In this paper we have shown that approximate symmetries can arise in a long-distance theory without any symmetry explanation in the underlying short-distance theory. Instead, even if symmetries are maximally broken at short distances, exponentially small couplings between different fields can result if they are "stuck" at slightly different points in extra dimensions. This 


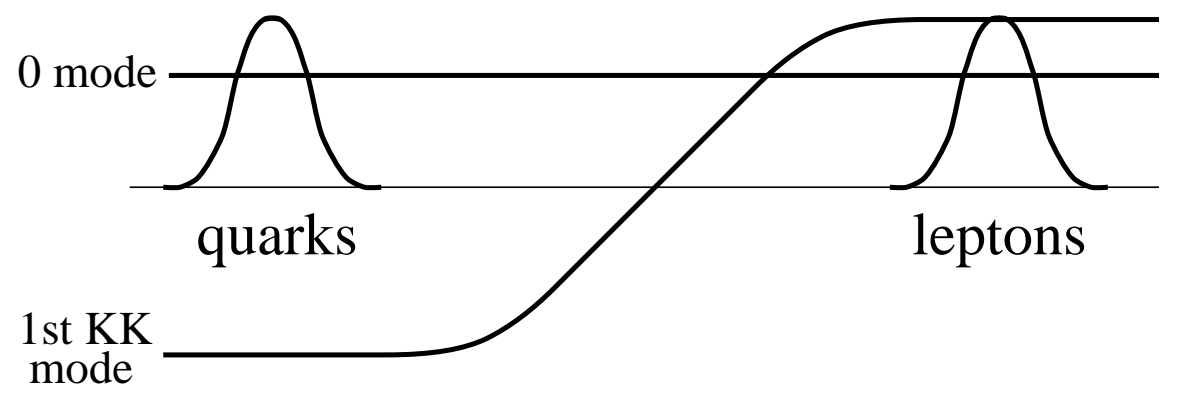

Figure 8: Since the wave functions of the usual SM gauge fields (zero modes) are flat in the extra dimensions, they have identical couplings to the quarks and leptons stuck at opposite ends of the wall, as required by gauge invariance. On the other hand, since the first $K K$ excitation has a non-trivial wave function, its couplings can be different. In particular, the product of quark and lepton gauge couplings to the first $K K$ excitation has the opposite sign as for the $S M$.

opens a new arena for model-building, where a specific arrangement of the fermions in extra dimensions, and not familiar flavor symmetries, determine the fermion mass hierarchy. Furthermore, proton decay can be elegantly disposed of, even in theories with the fundamental cutoff close to the $\mathrm{TeV}$ scale, if quarks and leptons are separated from each other by a factor of 10 larger than their size in the extra dimensions. If the effective size of this extra dimension or equivalently our wall thickness is close to the $\mathrm{TeV}$ scale, these ideas can be probed at the LHC and NLC. The smoking gun for our mechanism for would be the detection non-universality in the coupling of SM fermions to the KK excitations of the SM gauge fields. A detailed analysis of this non-universality could then be used to "map" the locations of the fermions in the extra dimensions. In closing we would like to emphasize that our mechanism of suppressing couplings from non-trivial wave functions is generically operative in higher dimensional theories with chiral fermions, as most such models obtain chiral matter from modes stuck to a defect in the higher dimensions. This defect may be a field theoretic domain wall in one 
extra dimension, a cosmic string in two extra dimensions, or a D-brane or orbifold fixed point ${ }^{\dagger \dagger}$ in a string model.

\section{Acknowledgements}

We thank Savas Dimopoulos, Lance Dixon, Gia Dvali, David Kaplan, John March-Russell, Gino Mirabelli, Shmuel Nussinov, Michael Peskin, Tom Rizzo and Eva Silverstein for discussions. Our work is supported by the Department of Energy under contract DE-AC03-76SF00515.

\section{References}

[1] N. Arkani-Hamed, S. Dimopoulos and G. Dvali, Phys. Lett. B429, 263 (1998).

[2] I. Antoniadis, N. Arkani-Hamed, S. Dimopoulos and G. Dvali, Phys. Lett. B436, 257 (1998).

[3] N. Arkani-Hamed, S. Dimopoulos and G. Dvali, hep-ph/9807344.

[4] N. Arkani-Hamed, S. Dimopoulos and J. March-Russell, hepth/9809124.

[5] See also E. Witten, Nucl. Phys. B471 (1996) 135; P. Horava and E. Witten, Nucl. Phys. B460 (1996) 506 and Nucl. Phys. B475 (1996) 94; J. Lykken, Phys. Rev. D54 (1996) 3693; G. Shiu and S.-H.H Tye, Phys. Rev. D58 (1998) 106007; R. Sundrum hep-ph/9805471 and hepph/9807348; Z. Kakushadze and S.-H.H. Tye, hep-th/9809147.

[6] K. Dienes, E. Dudas and T. Gherghetta, Phys. Lett. B436, 55 (1998); hep-ph/9806292.

\footnotetext{
${ }^{\dagger}$ The $e^{-r^{2}}$ suppression of Yukawa couplings between twisted sector fields at different orbifold fixed points has been known for some time, see e.g. [17].
} 
[7] N. Arkani-Hamed and S. Dimopoulos, hep-ph/9811353.

[8] D.B. Kaplan, Phys. Lett. B288 (1992) 342, hep-lat/9206013.

[9] P. Chen et. al., hep-lat/9812011.

[10] I. Antoniadis, Phys. Lett. B246 (1990) 377.

[11] S.C. Bennett, C.E. Wieman, hep-ex/9903022.

[12] The idea of localizing fermions on a wall in a higher dimensional space goes back to V. A. Rubakov and M.E. Shaposhnikov, Phys. Lett. B125 (1983) 136; which relied on the index theorem in soliton background R. Jackiw and C. Rebbi, Phys. Rev. D13 (1976) 3398; E. Weinberg, Phys. Rev. D24 (1981) 2669. Our discussion follows D.B. Kaplan and M. Schmaltz, Phys. Lett. B368 (1996) 44, hep-th/9510197.

[13] E. Witten, Nucl. Phys. B185 (1981) 513; Nucl. Phys. B202 (1982) 253.

[14] G. Dvali and M. Shifman, Phys. Lett. B399 (1997) 60.

[15] N. Arkani-Hamed and M. Schmaltz, hep-th/9812010.

[16] N. Arkani-Hamed, S. Dimopoulos, G. Dvali, and J. March-Russell, hep$\mathrm{ph} / 9811448$.

[17] L.E. Ibanez, Phys. Lett. B181 (1996) 269. 